\title{
Non-mastoidectomy Cochlear Implant Approaches: A Literature Review
}

\author{
Mohammad Waheed El-Anwar ${ }^{1} \quad$ Ahmed Shaker ElAassar $^{1} \quad$ Yaser Ahmad Foad $^{1}$ \\ ${ }^{1}$ Department of Otorhinolaryngology-Head and Neck Surgery, \\ Zagazig University, Zagazig, Egypt \\ Int Arch Otorhinolaryngol 2016;20:180-184. \\ Address for correspondence Mohammad Waheed El-Anwar, MD, \\ Department of Otorhinolaryngology Head and Neck Surgery, Zagazig \\ University, Zagazig 0020552309843, Egypt \\ (e-mail: mwenteg@yahoo.com).
}

\begin{abstract}
Introduction Posterior tympanotomy approach for cochlear implant $(\mathrm{Cl})$ surgery, has been the most commonly used worldwide with current $0.7 \%$ rate of facial nerve injury. Non-mastoidectomy $\mathrm{Cl}$ approaches include the suprameatal approach (SMA) and its modifications, the transcanal approach and its modifications and the pericanal approach for electrode insertion.

Objectives The objective of this study was to review the literature regarding nonmastoidectomy $\mathrm{Cl}$ approaches.

Data Synthesis A search was performed in the LILACS, MEDLINE, SciELO, PubMed databases and Cochrane Library in February 2015, and the key words used in the search were $\mathrm{Cl}$, SMA, transcanal approach, pericanal approach, or electrode extrusion. About 30 studies that met the criteria described in "Study Selection" were read in full. The studies showed 1014 patients that underwent $\mathrm{Cl}$ by SMA or its modifications, $266 \mathrm{Cl}$ patients treated by transcanal approach or its modifications, and 15 patients implanted by the pericanal approach. Reported complication with SMA was 99 (9.8\%) minor and 13 (1.3\%) major. With transcanal, there were 24 complications; 19 (7.1\%) minor and 5 (1.9\%) major. No post-operative complication was reported in pericanal approach. Studies showed no reported facial nerve paresis or paralysis in all non-mastoidectomy approaches.

\section{Keywords}

- cochlear implant

- mastoidectomy

- facial nerve

Conclusion Complications rates with non-mastoidectomy approaches are similar to those found in the mastoidectomy approach. Thus, non-mastoidectomy approaches may be an alternative in cases where the conventional mastoidectomy approach is difficult to perform. It would be helpful for $\mathrm{Cl}$ surgeons to become familiarized with these approaches.
\end{abstract}

\section{Introduction}

The posterior tympanotomy approach for cochlear implant (CI) surgery, described by House, has been the most commonly used $\mathrm{CI}$ surgery worldwide. So far, reports of facial nerve injury during facial recess surgery for $\mathrm{Cl}$ show a consistent rate of less than $1 \%$, with several studies reporting a rate of $0.7 \%{ }^{1-7}$ The vast majority of these are partial weaknesses of brief duration or delayed pareses of a temporary nature. Nonetheless, uneasiness of some surgeons dril- ling near the facial nerve has led to the development of alternate techniques for $\mathrm{Cl}^{1}$.

Some authors consider these alternative techniques (nonmastoidectomy approaches) critical and helpful in the presence of anatomic constraints, such as a small mastoid cavity, where there is greater difficulty in a facial recess approach. Additionally, there is a significant chance of aberrant facial nerve location in cases of labyrinthine dysplasia. Hoffman et al. ${ }^{2}$ reported facial nerve anomaly in $16 \%$ of patients with CI. Moreover, one third of these patients had either common received

April 13, 2015

accepted

May 17, 2015

published online

July 23, 2015
DOI http://dx.doi.org/

10.1055/s-0035-1558871. ISSN 1809-9777.
Copyright $(2016$ by Thieme Publicações License terms Ltda, Rio de Janeiro, Brazil

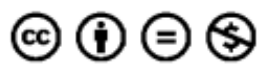


cavity malformation or hypoplastic cochlea with aberrant facial nerve course. ${ }^{2}$

Non-mastoidectomy $\mathrm{CI}$ approaches include the suprameatal approach (SMA) and its modifications, the transcanal approach and its modifications and the pericanal approach for electrode insertion.

\section{Methods}

We searched several medical databases, including LILACS, MEDLINE, SciELO, PubMed and the Cochrane Library in February 2015 to find out relevant articles.

We focused our review on studies involving the nonmastoidectomy approaches to $\mathrm{CI}$. Following Júnior et al., ${ }^{8}$ surgical complications were classified into major (if they require additional surgery or hospitalization), and minor, (when they resolve with outpatient treatment or even with no treatment). Major complications involve meningitis, flap necrosis, device failure, electrode extrusion, facial nerve paralysis and others; while the minor complications involve facial nerve stimulation, electrode migration, vertigo, tinnitus, and others. The major complications are less common. ${ }^{8}$

The authors analyzed the results of 12 studies that use the various non-mastoidectomy approaches to $\mathrm{CI}$, highlighting their importance as alternatives to the traditional mastoidectomy.

\section{Review of Literature}

\section{The Pericanal Approach for Electrode Insertion}

The pericanal approach is one of the alternative surgical techniques for $\mathrm{CI}$, which allows electrode insertion without performing a mastoidectomy. In this procedure, the electrode is placed directly through the EAC into the tympanic cavity. In brief, the procedure begins with a standard retroauricular incision, whereby the skin in the posterior EAC is lifted until the annulus of the TM is reached. The TM is retracted anteriorly, exposing the middle ear and its contents. A standard cochleostomy is performed slightly anterior to the round window. Then, using a $1.6 \mathrm{~mm}$ diamond burr, a vertical groove of $\sim 2 \mathrm{~mm}$ is created from the posterosuperior region of the bony EAC, above the incus body toward the lateral EAC, where a short superficial tunnel is created. A standard seat for the implant is created and fixed in the parietal bone; then, the electrode is led through the tunnel and the vertical bony groove into the tympanic cavity, where it is inserted into the cochleostomy. The electrode is fixed in place with glass ionomer cement and the groove is filled. The skin flap is replaced along the EAC and the ear is packed with gauze strips for 7-10 days. ${ }^{7}$

\section{The Suprameatal Approach (SMA) and Its Modifications}

Kronenberg et al. originally developed the Suprameatal approach to CI surgery in 1999, based on a blind atticotomy technique which was similar to a widely accepted approach to cholesteatoma. $^{3,4}$ The procedure involves raising a retroauricular skin flap and creating subperiosteal flap to drill a seat in the temporoparietal bone for the receiver-stimulator. Through the postauricular incision, a tympanomeatal flap is elevated. Once the chorda tympani nerve is identified, a 1$\mathrm{mm}$ groove is drilled in the scutum posterosuperior to the facial nerve and lateral to the incus until the incus short process is exposed. A blind tunnel is then drilled in the parietal bone just inferior to the temporal fossa bony plate and superior to the external auditory canal (EAC), aiming for the groove created in the scutum. Then, the cochleostomy is performed through the EAC, and the electrode is conducted through the suprameatal tunnel and the groove into the middle ear. It is then redirected to the cochleostomy in a near-vertical direction, and, again, redirected in an antero inferior direction in line with the scala. Later, Postelmans et al. introduced modifications to the original technique, in which the middle ear cavity is entered via an endaural tympanotomy rather than a postauricular incision, creating a subperiosteal tunnel between the seat for the receiver-stimulator and the suprameatal tunnel to protect the electrode. ${ }^{5}$

In 2004, Kronenberg and Migirov described 35 patients aged 1 to 67 years with $\sim 8$ months follow-up period that underwent $\mathrm{CI}$ by $\mathrm{SMA}^{9}$. Electrodes were inserted through Antro-inferior cochleostomy using several devices. They reported no surgical complications ${ }^{9}$.

Two years later, Kronenberg et al. ${ }^{10}$ described 188 patients who underwent SMA for $\mathrm{CI}$. They reported no facial nerve injuries or cerebrospinal fluid (CSF) leaks and no cases of electrode extrusion into the EAC. There were, however, 4 perforations of the tympanic membrane (TM) (2\%) where the scutum was drilled. ${ }^{10}$

In 2004, there were 255 reported cases of patients who underwent CI by SMA, where 185 were in Tel Aviv and 70 in Vienna. Electrode insertion was through Antro-inferior cochleostomy with Combi 40 and Combi 40+ (MEDEL) devices and Nucleus and Clarion electrode devices. There were no surgical complications reported $^{11}$.

In China, 2008, Yin et al. ${ }^{12}$ described 45 patients who underwent CI by SMA (a total 47 ears). Patients average age was 5.7 years; 29 were male and 16 female, undergoing a follow-up period ranging from 1 to 20 months. Electrodes were inserted through Antro-inferior cochleostomy. Of the implants, 42 were Med-El C40+ while the other five were Clarion 90k. They reported that 38 patients with Med-El implants had all electrode pairs entirely inserted in the cochlea; one patient with cochlear ossification, however, had 9 pairs of electrodes inserted, and another patient with profound cochlear dysplasia had 8 pairs of electrodes inserted. All electrode pairs were inserted in the five patients using a Hires 90KTM implant. During surgery, a "gusher" occurred in one patient with profound cochlear dysplasia, and was treated by sealing the cochleostomy using a small piece of teamporalis muscle and biological glue. The electrode was inserted lateral to the chorda tympani in one patient because the chorda tympani had adhered to the body of the incus. ${ }^{12}$ Three patients with large vestibular aqueduct syndrome 
(LVAS) and one patient with profound cochlear dysplasia had postoperative vestibular dysfunction symptoms such as vertigo, nausea, and vomiting. Among the 30 patients who were followed for more than 6 months, 12 had open set speech perception and could effectively communicate with others; 14 patients learned to speak short sentences even if not clearly; and 4 patients could only say single words. ${ }^{12}$

In 2012, Tange and Tange ${ }^{13}$, from the Netherlands, reported 260 cases with mean age 39.6 years who underwent $\mathrm{Cl}$ by SMA. The electrodes were inserted through Antro-inferior cochleostomy. The study used four different types of cochlear implants: the Nuc1eus 24-Countour, the Nuc1eus 24-Countour Advance with Softip, Advanced Bionics HiFocus Helix and Medel Sonata. The authors found that the minor complication rate was $23 \%$, which consisted of tinnitus (7.2\%), postoperative vertigo (5.2\%), TM perforation (1.3\%), hematoma (1.3\%), and other causes (3.8\%). In one patient (otosclerosis), mild facial nerve stimulation was found and could be managed by switching off several electrodes. All of the five patients that reported minor complications were successfully treated. On the other hand, all five cases of major complications (3\%) developed postoperatively. The major complications consisted of two cases of implant extrusion due to wound infection, a fausse route of electrode 11 , a misdirected electrode in severe otosclerosis, and a case of explanation due to psychiatric illness and pain sensations. All these five cases were re-implanted and had successful results thereafter, except for the patient with the psychiatric illness. This patient continues to complain, in spite of a perfectly functioning device and fully healed wound. One device failure in one patient (speech recognition had declined one year after implantation) required explanation and re-implantation. ${ }^{13}$

In 2010, Guevara et al. ${ }^{14}$ described 23 patients who underwent $\mathrm{CI}$ by SMA in France, of which 13 were female and 10 were male. Patient ages ranged from 8 months to 63 years with mean follow up duration of 22 months. Electrodes were inserted through Antro-inferior cochleostomy. In the first patient, the anterior facial recess hole was done too high; thus, the incus had to be removed to allow the electrode through. Although the tympanomeatal flap had been elevated without incising the posterior wall of the external auditory skin, no TM was observed. ${ }^{14}$

In the same year, Postelmans et al. from the Netherlands described 108 patients who underwent $\mathrm{CI}$ by SMA, with mean age 39.6 years and mean follow-up period of 25.7 months. Electrode insertion was through Antro-inferior cochleostomy using Nucleus 24 Countour, Nucleus 24 Countour Advance with Softip, and Advanced Bionics HiFocus Helix devices. The study reported an overall major complication rate of 3.7\% (4 out of 107 patients) that developed postoperatively. Results included extrusion of the implant due to wound infection $(n=2)$, a wrong route for the electrode $(n=1)$, and device failure $(n=1)$. The minor complication rate was $23.4 \%$ ( 25 out of 107 patients). ${ }^{5}$

In 2012, Zernotti et al ${ }^{15}$ described 80 cases of $\mathrm{CI}$ by SMA in Spain and England, in which electrodes were inserted through Antro-inferior cochleostomy. The authors reported that one patient suffered from a wound infection, four suffered from vestibular complications and two suffered from electrode displacement. As for major complications, there was only one case documented, in the form of device failure. $^{15}$

Therefore, of the 1014 patients with SMA CI whose cases have been published, there were $99(9.8 \%)$ minor and 13 (1.3\%) major complications reported and no reports of facial nerve paresis or paralysis ( $\mathbf{-}$ Table $\mathbf{1}$ ).

\section{The Transcanal (Veria) Approach}

Transcanal operation can be summarized by the following steps: 1- endaural or retroauricular approach to the middle ear with elevation of a standard tympanomeatal flap, 2inspection of the middle ear anatomy (cochlea, fallopian canal, round window niche), 3- straightening of the posterosuperior bony canal wall, 4- cochleostomy, 5- drilling the suprameatal hollow, 6- drilling the transcanal wall direct tunnel with alignment to the cochleostomy, 7- extension of the skin incision and preparation of the skin flaps and subperiosteal flap, 8- creating the bed and fixing the device, 9 - insertion of the electrode, 10- manipulation of the excess electrode in the suprameatal hollow, and closing. ${ }^{6}$

In Spain, 2008, Slavutsky and Nicenboim ${ }^{16}$ described 10 patients who underwent $\mathrm{CI}$ by the transcanal approach, whereby electrodes were inserted via the round window. The group's mean age was 33.1 years and consisted of 3 men and 7 women, going through a follow-up period that ranged from 3 to 18 months. The authors reported that the ten devices had been working properly and results were as expected for all patients, without complications. ${ }^{16}$

In 2009, from the Kingdom of Saudi Arabia (KSA), Taibah ${ }^{17}$ described 131 patients who underwent $\mathrm{CI}$ by the transcanal approach, with electrodes inserted via Antro-inferior

Table 1 The complication rate differences between transcanal and suprameatal approaches (SMA)

\begin{tabular}{|l|l|l|l|l|}
\hline & SMA & Transcanal & Chi-square & P value \\
\hline Total number of Cl & 1014 & 266 & & \\
\hline Total complications & $112(11 \%)$ & $24(9 \%)$ & 0.908 & 0.34 NS \\
\hline Major complications & $13(1.3 \%)$ & $5(1.9 \%$ & 0.543 & $0.46 \mathrm{NS}$ \\
\hline Minor complications & $99(9.8 \%)$ & $19(7.1 \%)$ & 1.843 & $0.17 \mathrm{NS}$ \\
\hline Electrode extrusion & $8(0.79 \%)$ & $5(1.88 \%)$ & 2.494 & $0.114 \mathrm{NS}$ \\
\hline
\end{tabular}

Abbreviations: Cl, cochlear implant; NS, non-significant; SMA, suprameatal approach. 
cochleostomy. The group was comprised of 115 children, with ages ranging from 10 months to 14 years, and 16 adults, with ages ranging from 24 to 58 years, with a male-to-female ratio of $\sim 1: 1$. Follow-up duration ranged from 2 to 46 months. The author reported five cases of small TM perforations after acute otitis media; these TM perforations healed spontaneously after medication in four children. One adult needed myringoplasty. Furthermore, one child with bilateral CI who had a polyp in the right meatus of the EAC did not respond to topical antibiotics. The child underwent a re-exploration of the post-auricular area under general anesthesia, due to fear of possible electrode extrusion. The polyp was removed and the EAC bone had completely healed with no extrusion of the electrode array. In two children, an unexpected infection developed around the receiver in the temporal region. ${ }^{17}$

Mostafa et al., ${ }^{18} 2014$, described 125 cases in Egypt who underwent $\mathrm{CI}$ by the modified transcanal approach. The study group's mean age was 3.4 years, male-to-female ratio was 2.1:1, and follow-up period ranged from 18 to 50 months. Electrode insertion was via the round window and the devices used were Cochlear (Nucleus Freedom), MedEl (Sonata), Advanced Bionics (His res $90 \mathrm{~K}$ ), and Neurelec (Saphyr). They reported 5 gushers; 2 patients with EVAS (enlarged vestibular aqueduct syndrome) and 3 patients with IP2-type malformations. Six patients suffered chorda tympani injury; two of them complained of loss of taste on the ipsilateral tongue. Two cases had a small TM perforation; both were eventually grafted uneventfully, without compromising device activation. Two patients showed electrode exposure, one of which required revision. One patient had severe infection with extrusion of the device one year after its successful implantation. ${ }^{18}$

In sum, of the reported 266 cases of transcanal CI, 24 suffered complications, of which 19 (7.1\%) were minor and 5 (1.9\%) were major, with no reported facial palsy or paralysis (-Table 1).

The differences in complication rates between transcanal and SMA is non-significant (-Table $\mathbf{1}$ ).

\section{The Pericanal Approach for Electrode Insertion}

Hausler $2002^{19}$, in Thailand, described 15 patients with an age range of 10 to 48 years, ( 8 females and 7 males), with follow-up period ranging from 6 to 24 months, who underwent CI with pericanal insertion technique. Combi 40 (MEDEL) was used in 14 patients and a Nucleus CI24M in one patient. The author found that in one patient with a narrow space between the long incus process and the malleus handle, the incus had to be luxated and displaced posteriorly to allow easier passage of the electrode through the superior part of the tympanic cavity. In four patients, small lacerations of the skin of the EAC occurred during elevation of the posterior tympano-meatal flap. The EAC healed without problems in all cases. In two patients, a small postero-inferior lesion of the TM had to be closed at the end of surgery with a mini-graft of adipose tissue. Despite some operative difficulties and problems, there was no reported post-operative complication.

\section{Discussion}

From the literature analyzed, we found that among the 1014 patients who underwent CI by SMA, 112 had surgical complications (11\%), 13 (1.3\%) had major complications, and 99 (9.8\%) had minor complications.

The major complications comprised four cases $(0.39 \%)$ that had electrode extrusion due to infection, four cases that had misdirected electrode, four cases of device failure, and a case that required exploration due to psychiatric illness and pain sensations.

On the other hand, among the 266 cases that underwent $\mathrm{CI}$ by the transcanal approach, 24 (9\%) had surgical complications, 5 had major complications (1.9\%), and 19 had minor complications (7.1\%).

The major complications were three cases $(0.3 \%)$ of electrode extrusion due to infection and two cases (0.2\%) with electrode exposure.

Hashemi et al., ${ }^{19}$ in a study of 181 cases that underwent the PTA technique, reported major surgical complications in $2.66 \%$ of them. The rate of device failure was $2 \%$; therefore, the unwanted outcomes requiring surgery were $4.66 \%$, while $10 \%$ reported minor complication rate. ${ }^{19} \mathrm{Xu}$ et al., ${ }^{20}$ in a metaanalysis study of 799 cases, reported no statistically significant difference in major and minor complications between the two approaches, except for facial nerve and chorda tympani injuries, favoring SMA. ${ }^{20}$ Migirov et al. ${ }^{21}$ operated 300 cases of $\mathrm{CI}$ and reported a rate of $3 \%$ major complications and $25.7 \%$ minor complications. ${ }^{21}$ Júnior et al., ${ }^{8}$ in a study of 250 cases, reported 13 cases (5.2\%) of major postoperative complications and 20 cases ( $8 \%$ ) of minor complications. ${ }^{8}$

Kevin et al. ${ }^{22}$ reported that the most common reasons for revision were device malfunction. They found that $7.8 \%$ had device malfunction, out of 805 cases operated by both approaches. Fayad et al. ${ }^{23}$ reported $7 \%$ postoperative flap breakdowns with implant extrusion. Ajalloueyan et al. ${ }^{24}$ reported $1 \%$ of device malfunction in 262 patients that underwent $\mathrm{CI}$.

Ajalloueyan et al. ${ }^{24}$ reported that, out of the 262 patients on which the PT approach was used, two had wound infection $(0.8 \%)$ post $\mathrm{Cl}^{24}$. Junior et al. ${ }^{8}$ reported that 5 cases (2\%) had wound infection post $\mathrm{CI}$, out of 250 cases by PT approach.

Migirov et al. ${ }^{21}$ reported that two cases had facial nerve paralysis ( $0.66 \%$ ), out of 300 cases operated by the PT approach. Using the same operative approach, Ajalloueyan et al. ${ }^{24}$ reported that $1 \%$ of the 262 patients operated had facial nerve paralysis. Current reports of facial nerve injury during facial recess surgery for $\mathrm{CI}$ show a consistent rate of less than $1 \%$, with several studies reporting a rate of $0.7 \%{ }^{25-29}$ Nonetheless, none of these techniques led to facial nerve paralysis. The study showed that the non-mastoidectomy approaches could avoid facial nerve and chorda tympani injuries in patients with $\mathrm{CI}$ with low rates of other complications.

After analyzing the literature, we are convinced that nonmastoidectomy (SMA, tanscanal, pericanal) approaches for $\mathrm{CI}$ are significantly faster, easier and safer in that which pertains to facial nerve, when compared to the PT approach. In over one thousand published cases of SMA, total incidence of complications were $11 \%$. Furthermore, major complications were 
detected in only $1.3 \%$ with transcanal, while a total of $9 \%$ reported no complications with the permeatal approach. These complications rates are similar to those found in the mastoidectomy approach, albeit with the additional advantage of shorter duration of surgery, anesthesia, recovery and hospital stay.

This study also reveals that electrode fixation was not a matter of concern in the techniques analyzed, given that the rate of electrode extrusion in over 1000 patients was $0.79 \%$ in SMA and $1.88 \%$ for the transcanal approach. Junior et al. ${ }^{8}$ reported one case $(0.4 \%)$ of electrode exclusion out of 250 cases that underwent $\mathrm{CI}$ by the PA approach, and Raghunandhan et al. ${ }^{30}$ reported another single case $(0.3 \%)$, out of 300 cases that underwent $\mathrm{CI}$ by the PT approach.

\section{Final Comments}

Alternatively, non-mastoidectomy approaches are valid in cases where conventional PT is difficult to perform. Signs that may indicate this method include narrow facial recess, anteriorly located facial nerve, ossified cochlea (due to the promontory is more exposed in details in non-mastoidectomy approaches better than classic approaches) and severelycontracted mastoid. Therefore, the authors consider it helpful and believe it should be mandatory that $\mathrm{CI}$ surgeons become familiar with these approaches.

\section{Conflict of Interest and Financial Disclosure}

The authors declare that there are no conflicts of interest and that no financial support or interest is vested in this study.

\section{References}

1 Choi JE, Jang JY, Cho YS. Cochlear implantation using a suprameatal approach in a case of severely contracted mastoid cavity. Korean J Audiol 2014;18(3):144-147

2 Zeitler DM, Balkany TJ. Alternative approaches to cochlear implantation. Operative Techniques in Otolaryngology 2010;21(4):248-253

3 Kronenberg J, Migirov L, Dagan T. Suprameatal approach: new surgical approach for cochlear implantation. J Laryngol Otol 2001; 115(4):283-285

4 Kronenberg J, Baumgartner W, Migirov L, Dagan T, Hildesheimer M. The suprameatal approach: an alternative surgical approach to cochlear implantation. Otol Neurotol 2004;25(1):41-44, discussion 44-45

5 Postelmans JT, Grolman W, Tange RA, Stokroos RJ. Comparison of two approaches to the surgical management of cochlear implantation. Laryngoscope 2009;119(8):1571-1578

6 Kiratzidis T, Arnold W, Iliades T. Veria operation updated. I. The trans-canal wall cochlear implantation. ORL J Otorhinolaryngol Relat Spec 2002;64(6):406-412

7 Häusler R. Cochlear implantation without mastoidectomy: the pericanal electrode insertion technique. Acta Otolaryngol 2002; 122(7):715-719

8 Lima Júnior LR, Rodrigues Júnior FdeA, Calhau CM, Calhau AC, Palhano CT. Postoperative complications in implanted patients in the Cochlear Implant Program of Rio Grande do Norte-Brazil. Braz J Otorhinolaryngol 2010;76(4):517-521

9 Kronenberg J, Migirov L. Application of minimal access surgery for cochlear implantation using suprameatal approach. Int Congr Ser 2004;1273:111-114
10 Kronenberg J, Migirov L. The suprameatal approach: an alternative surgical technique for cochlear implantation. Cochlear Implants Int 2006;7(3):142-147

11 Baumgartnera WD, Jappela A, Kronenberg J, Hamzavia J, Freia KM, Stach M. Cochlear implantation without mastoidectomy: the Vienna experience. Int Congr Ser 2004;1273:122-124

12 Yin S, Chen Z, Wu Y, et al. Suprameatal approach for cochlear implantation in 45 Chinese children. Int J Pediatr Otorhinolaryngol 2008;72(3):397-403

13 Tange RA. Modifications on the Alternative Method for Cochlea Implantation, Cochlear Implant Research Updates, Dr. Cila Umat (Ed.) 2012, 2-27

14 Guevara N, Bailleux S, Santini J, Castillo L, Gahide I. Cochlear implantation surgery without posterior tympanotomy: can we still improve it? Acta Otolaryngol 2010;130(1):37-41

15 Zernotti ME, Suárez A, Slavutsky V, Nicenboim L, Di Gregorio MF, Soto JA. Comparison of complications by technique used in cochlear implants. Acta Otorrinolaringol Esp 2012;63(5):327-331

16 Slavutsky V, Nicenboim L. Preliminary results in cochlear implant surgery without antromastoidectomy and with atraumatic electrode insertion: the endomeatal approach. Eur Arch Otorhinolaryngol 2009;266(4):481-488

17 Taibah K. The transmeatal approach: a new technique in cochlear and middle ear implants. Cochlear Implants Int 2009;10(4): 218-228

18 Mostafa BE, Ezzat WF, El Mogui A. The Modified Transcanal Approach for Cochlear Implantation: Technique and Results. Advances in Otolaryngology 2014http://dx.doi.org/10.1155/2014/509703

19 Hashemi B, Bayat AL, Kazemei T. Surgical Complications of Cochlear Implantation. Iran J Med Sci 2010;35(1):53-55

20 Xu BC, Wang SY, Liu XW, et al. Comparison of complications of the suprameatal approach and mastoidectomy with posterior tympanotomy approach in cochlear implantation: a meta-analysis. ORL J Otorhinolaryngol Relat Spec 2014;76(1):25-35

21 Migirov L, Dagan E, Kronenberg J. Surgical and medical complications in different cochlear implant devices. Acta Otolaryngol 2009; 129(7):741-744

22 Brown KD, Connell SS, Balkany TJ, Eshraghi AE, Telischi FF, Angeli SA. Incidence and indications for revision cochlear implant surgery in adults and children. Laryngoscope 2009; 119(1):152-157

23 Fayad JN, Baino T, Parisier SC. Revision cochlear implant surgery: causes and outcome. Otolaryngol Head Neck Surg 2004;131(4): 429-432

24 Ajalloueyan M, Amirsalari S, Yousefi A, Raeesi MA, Radfar S, Hassanalifard M. Report of Surgical Complications in a Series of 262 Consecutive Pediatric Cochlear Implantations in Iran. Iran J Pediatr 2011;21(4):455-460

25 Cohen NL, Hoffman RA, Stroschein M. Medical or surgical complications related to the Nucleus multichannel cochlear implant. Ann Otol Rhinol Laryngol Suppl 1988;135:8-13

26 Hoffman RA, Cohen NL. Complications of cochlear implant surgery. Ann Otol Rhinol Laryngol Suppl 1995;166:420-422

27 Fayad JN, Wanna GB, Micheletto JN, Parisier SC. Facial nerve paralysis following cochlear implant surgery. Laryngoscope 2003;113(8):1344-1346

28 Hehar SS, Nikolopoulos TP, Gibbin KP, O’Donoghue GM. Surgery and functional outcomes in deaf children receiving cochlear implants before age 2 years. Arch Otolaryngol Head Neck Surg 2002; 128(1):11-14

29 Kandogan T, Levent O, Gurol G. Complications of paediatric cochlear implantation: experience in Izmir. J Laryngol Otol 2005;119(8):606-610

30 Raghunandhan S, Kameswaran M, Anand Kumar RS, Agarwal AK, Hossain MD. A study of complications and morbidity profile in cochlear implantation: the MERF experience. Indian J Otolaryngol Head Neck Surg 2014;66(1, Suppl 1):161-168 